\author{
Christian Beer $^{\mathrm{a}}$ \\ Klaus von Ammon ${ }^{\mathrm{b}}$
}

In der Schweiz liegen seit 1926 epidemiologische Daten vor, dass die Pollenallergie bei Erwachsenen innerhalb von 70 Jahren von unter $1 \%$ auf über $12 \%$ angestiegen ist. Bei Kindern und Jugendlichen bewegt sich die Prävalenz seit Mitte der 1990er-Jahre unverändert um $15 \%$. Gründe für die Zunahme der Pollenallergie liegen in der Haltung von Haustieren, der zunehmend besseren Isolation von Wohnungen und Häusern und der allgemeinen Luftverschmutzung. Städter leiden häufiger an allergischen Atemwegserkrankungen als Personen, die auf dem Land leben [1].

Die schulmedizinischen Abklärungen (Prick-Test und Radio-AllergoSorbent-Test (RAST)) und Behandlungen (Antihistaminika, Steroide, Desensibilisierung) sind teuer, aufwändig und mit Nebenwirkungen behaftet. Somit drängt sich die Homöopathie als einfache, ungefährliche, kostengünstige, effiziente und alternative Therapie geradezu auf. Methodische Grundlage bleiben die gezielte Anamnese nach heuschnupfenspezifischen Symptomen sowie die klinische Untersuchung und Beobachtung. Es wird zwischen der saisonalen bzw. akuten Behandlung und der chronischen bzw. endgültigen Sanierung der Pollinosis unterschieden. Während bei der Akuttherapie nur diejenigen Symptome, die sich während der akuten Phase einstellen, wichtig sind, müssen bei der chronischen Behand-

\footnotetext{
${ }^{a}$ Allgemeine Medizin FMH und FA für Homöopathie SVHA, Dürrenroth, Schweiz ${ }^{b}$ Spezialarzt FMH und FA für Homöopathie SVHA, Stäfa, Schweiz
}

\title{
Die homöopathische Behandlung des Heuschnupfens in der Praxis
}

lung zusätzlich alle individuellen Symptome des Patienten berücksichtigt werden, um das Grundmittel zu finden.

Die klassische Homöopathie versucht mittels genauer Anamnese und Beobachtung ein Arzneimittel nach dem individuellen Symptomenbild des Kranken auszuwählen. Die Erhebung der Beschwerden sollte möglichst nicht vor Ablauf der ersten Erkrankungswoche vorgenommen werden, um präzise Angaben zu erhalten. Es ist entscheidend, durchgehende Symptome sowie das dem jeweiligen Patienten Spezifische des eigenen Heuschnupfens zu erfassen. Es ist nützlich, dem Patienten einen Handzettel mit folgenden Fragen abzugeben («<» gilt für Verschlimmerung, «>» für Besserung der Beschwerden):

- Nase: Fliessschnupfen (mild, scharf, Farbe, Geruch, Seite) oder Verstopfung (Krusten); Geruchssinn (überempfindlich, schwach); Modalitäten, z.B. < Niesen, < Gerüche, $<$ Temperaturwechsel, > kalte Umschläge, > Schnupfen fliessend, $>$ Reiben;

- Augen: Lokalisation (Seite, Augenwinkel, Lidränder), Tränenfluss (mild, scharf, brennend), Fremdkörpergefühl, Lidschwellung oben/ unten, Lidfläche innen, Aufgedunsenheit um Augen herum, dunkle Augenringe; Modalitäten, z.B. $<$ Licht, $</>$ Schliessen der Augen, $</>$ Blinzeln, $>$ kalte Umschläge;

- Ohren: Jucken;

- Mund, Gaumen, Rachen: Kratzen, Trockenheit, Jucken;

- Begleitsymptome: Atmung, Husten, Stimmung [2].
Diese Symptome werden gesammelt, nach ihrer Wichtigkeit geordnet (hierarchisiert) und diejenigen Arzneien ermittelt (repertorisiert), die bei Gesunden solche Symptome hervorgerufen haben. Als Repertorisationsmethoden bewähren sich in der Praxis die Programme Amokoor [3] und Bönninghausen [4]. Hilfreich ist auch Klaus-Henning Gypsers Büchlein «Grundzüge der homöopathischen Heuschnupfenbehandlung» [5], in dem sich der Autor auf 20 klinisch geprüfte Arzneien beschränkt und eine kleine Materia medica mit entsprechendem Repertorium aufstellt. Folgende, oft «kleine» Mittel (selten verordnet und mit eng umschriebenem Symptomenbild) gelangen zur Auswahl: Allium cepa (Küchenzwiebel), Arsenicum album (Arsen), Arum triphyllum (Zehrwurzel), Arundo (Pfahlrohr), Bromium (Brom), Carbo vegetabilis (Holzkohle), Euphrasia (Augentrost), Jodum (Jod), Kalium jodatum (Kaliumjodid), Lachesis (Gift der Buschmeisterschlange), Nux vomica (Brechnuss), Pulsatilla (Küchenschelle), Ranunculus bulbosus (Knolliger Hahnenfuss), Sabadilla (Läusesamen), Sanguinaria (Kanadische Blutwurzel), Sinapis nigra (Schwarzer Senf), Squilla maritima (Meerzwiebel), Sticta pulmonaria (Lungenmoos) (Abb. 1), Teucrium (Gamander), Wyethia (Wyethia).

Die Behandlungsergebnisse Gypsers sind ermutigend: Saison 2001: Patientenzahl (kurativ Behandelte in Klammern) 48 (34; 70,8\%); 2002: 51 (39; 76,5\%); 2003: 24 (18; 75\%). Frei [2] fand bei 103 erfolgreich behandelten Heuschnupfenpatienten folgende Häufigkeitsverteilung der Arzneimittel: Sul-

\section{KARGER}

Fax +49 7614520714

E-Mail Information@Karger.de

www.karger.com
(๑) 2010 S. Karger GmbH, Freiburg
Dr. med. Christian Beer

Oberwaldstrasse 8, 3465 Dürrenroth, Schweiz

Tel. +41 62 96414-85, Fax -87

cbeer@sunrise.ch 
Abb. 1. Sticta pulmonaria (Lungenmoos).

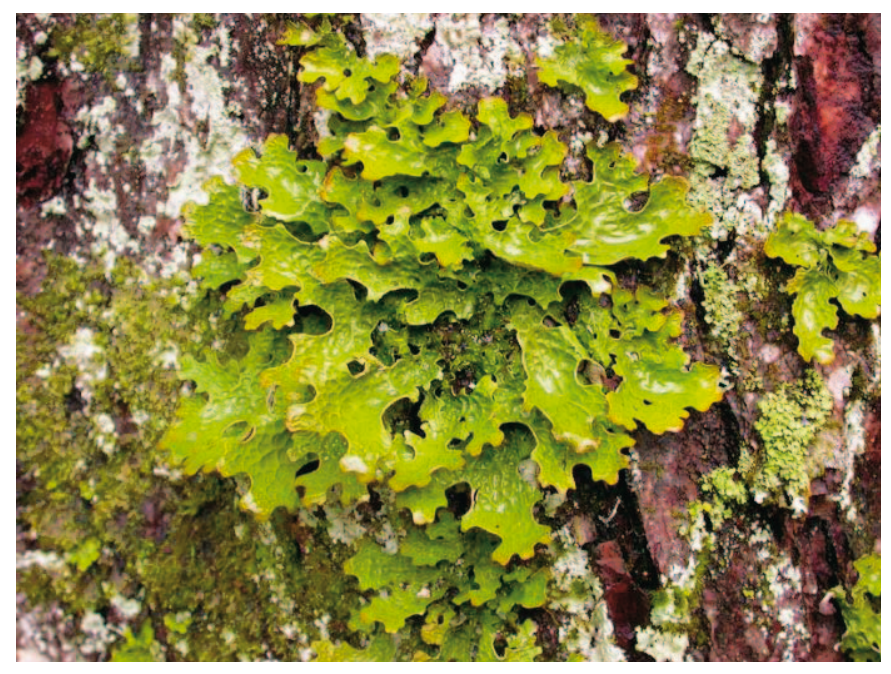

fur: 21, Pulsatilla: 16, Arsenicum album: 12, Calcium carbonicum: 12, Nux vomica: 10, Phosphor: 6, Sepia: 6, Silicea: 5, Lycopodium: 4, Euphrasia: 3, Rhus toxicodendron: 2; Allium cepa, Belladonna, Bryonia, China officinalis (Chinarinde), Staphisagria, Sticta pulmonaria je 1.

Bei einer solchen Akutbehandlung besteht das Ziel zumindest in einer Linderung oder einem schnellen Rückgang der Beschwerden. Oft ist damit während der Pollensaison der «Fall gelöst». Schwieriger wird es, wenn im nächsten Jahr die gleichen Beschwerden wieder auftreten. Gibt es bei einer neuen Fallaufnahme keine neuen Gesichtspunkte, kann die gleiche oder nächsthöhere Potenz derselben Arznei verordnet werden. Haben sich die Befunde geändert oder gibt es neue Symptome, muss eine neue Repertorisation vorgenommen und wahrscheinlich ein anderes Mittel verordnet werden. Führt diese Behandlung nicht zum Ziel oder handelt es sich um eine chronische Erkrankung, muss eine sog. grosse Fallaufnahme weitere, für die erkrankte
Person charakteristische Symptome, besonders in ihrem gegenwärtigen Zustand, aufspüren. Dabei sind die für einen (typischen) Krankheitsverlauf eines Heuschnupfens ungewöhnlichen, die erkrankte Person aber individuell auszeichnenden Symptome von besonderer Wichtigkeit [6]. Bei der Auswertung dieser weiterführenden Symptome kann man eine Arznei finden, die bei wiederholter Gabe ein erneutes Auftreten des Heuschnupfens deutlich abschwächt oder nach 2-3 Jahren unwahrscheinlich macht. Zudem wird ein solches «Grundmittel» die Entwicklung eines Bronchialasthmas verhindern, was sonst häufig bei atopischer Disposition oder nicht bzw. unzureichend behandeltem Heuschnupfen vorkommt. Idealerweise sollte diese Arznei auch andere Erkrankungen oder Störungen des Patienten bessern oder nach wiederholter Gabe ausheilen.

Heuschnupfen ist auch in der homöopathischen Forschung untersucht worden, ohne dass deren sehr gute Ergebnisse praktische Bedeutung für die klassische, individuell verordnende
Homöopathie gewonnen hätte: Der (isopathische) Doppelblindversuch gegen Placebo von Reilly und Taylor [7] hat international mehr aufgrund der strengen Durchführung als wegen des statistisch signifikanten Ergebnisses zugunsten von Pollen (C30) Beachtung gefunden. Bis heute sind nämlich keine weiteren Anwendungen von potenziertem Pollen in grösserem Stil bekannt geworden. Wiesenauer [8] hat 1980 und 1982 die Wirkung von Galphimia glauca D4 und D6 bei 86 und 164 Patienten mit einem Therapieerfolg von 83 und $72 \%$ nach 5-6 Wochen in Doppelblindversuchen nachgewiesen. Klinische Nachuntersuchungen nach je 1 Jahr ergaben Ergebnisse von $90 \%(\mathrm{n}=81)$ und $70 \%(\mathrm{n}=160)$ Beschwerdefreiheit oder deutlicher Linderung. Galphimia glauca hat in der klinisch-organotropen Homöopathie, vor allem in Deutschland, eine gewisse Bedeutung in der Anwendung von niedrigen Potenzen erhalten.

\section{Literatur}

1 Dürr C, Heimgartner S, Gehrig R, Caversaccio M, Helbling A: Pollenallergie: Klinische Aspekte. Teil 1. Schweiz Med Forum 2008;8:253257.

2 Frei H, Beer C: Workshop Rhinitis und Konjunctivitis allergica. SAHP. Skriptum GK 11/ 2009.

3 Steiner U: Amokoor Version 9.0. Thun, Praxidata, 1992.

4 Bönninghausen CM, Gypser KH: Bönninghausens Therapeutisches Taschenbuch, revidierte Ausgabe, ed 3. Stuttgart, Sonntag, 2000.

5 Gypser KH: Grundzüge der homöopathischen Heuschnupfenbehandlung. Glees, W. Gypser, 2005.

6 Dahler J, Teut M, Lucae C: Homöopathie bei Heuschnupfen. Stuttgart, Hippokrates, 2008.

7 Reilly D, Taylor M: Is homeopathy a placebo response? Lancet 1986;2:881-886.

8 Wiesenauer M: Allergische Rhinitis. Z Allgemeinmed 1986;62:388. 